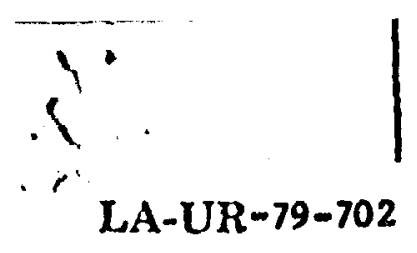

AA-UR-79-702

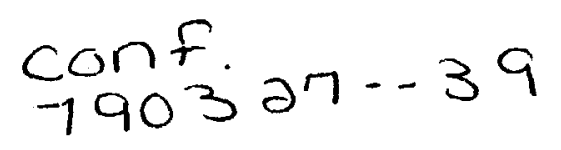

MASTER
AUTHOS(́́): D(aniel) W(ebster) Hudgings, D(avid) A(1lison)
Clark, H(oward) C(arnes) Bryant, C(harles)
$A(11$ en) Frost, J(oey) B(urke) Donahue.
R (obert) W(ray) Hamn, M(ichael) W(alford)
McNaughton, J(chn) C(harles) Pratt, M(arrianne)
$E$ Hamm, and $M(\operatorname{ary}) A(n n)$ Yates-Willians

\section{SUBMITTED TO:}

Conference Proceedings of the 1979

Particle Accelerator Conference,

San Francisco, California, March 12-14, 1979

By acceptance of this article for publication, the publisher recognizus the Government's (license) rights in uny copyright and the Government and its authorized representutives huve unrestricted right to reproduce in whole or in part anid article under any copyright secured by the publisher.

The los Al:umos Scientific l.uboritory requests that the publisher identify this article ns work performed under the auspices of the USHEDA. 
1 TIM-RESOLVED BEAM ENERGY MEASUREMENTS AT LAMPY"

'D. A. Clark, H. C. Bryant, and C. A. Froet and J. B. Donahue, M. E. Hem R. W. Hanm, D. W. Hudginga,

M. W. Mclaughton, J. C. Pratt, and M. A. Yaten-Will ians

Los Alemon Scientific Laboratory, LOS Alamos, MM 87545

\section{Abstract}

A darrow atomic photodetachwent resonance is used to messure the LAMPE beaw energy. Energy and time resolution are adequate to permit the use of this wethod in studying transient changes in accelerated be:su energy.

\section{Introduction}

The high current proton storage ring (PSR) now being designed ${ }^{2}$ at the Los Alemos Scientific Laboratory will accumulate 800-MeV protons from the LAMPF linac and deliver thea in very intense short pulses to a heavy metal target at the Weapons Neutron Research (WNR) Facility. Figure I hows the LAMPE pulse structure, which consists of macropulses of half willisecond duration repeated at a 120 pulge per second rate. Each macropulse consists of 105 micropulses of $<i C^{-10}$ second duration containing $5 \times 10^{8}$ protons or nagative hydrogen $\left(H^{*}\right)$ ions.

At present, when nanosecond neutron pulses are required at WNR, single mieropul ses of protons are selected from the LANPF macropulses and delivered to the neutron production target. Much more intense nanosecond-long pulses (containing 1011 protons)

will be produced by the PSA operating in the storage mode illustrated in $\mathrm{rig}$. 2. The $\left(\mathrm{H}^{-}\right)$ion micropulses accelerated at 60 nanosecond intervals by the linac will be accumulated in the PSR as six citculating proton bunches separated in the ring by 60 nanosecond spacings. The $\mathrm{H}^{-}$ions are injected for 200 turns $(72 \mu)$, by means of a two-step charge changing method. $\{$ The bunches are maintained during the accumulation process by $\$ 500$ MHz rf buncher. After full intensity is reached they are ejected towards the WhR target at intervals of 1.4 miliisecond.

In this mode of operation the energy acceptance of the PSR is quite soall because the proton transit $t$ ime around the ring must be. $t$ or near an integer multiple of the 5 nanosecond micx pulse spacing to properly synchronize newly injected $\mathrm{H}^{-}$ions with protons al ready in the ring. This requirenent on the circulation period fixes the LANPP beam energy (and energy

Work supported by U. S. Department of Energy *hiniversity of New Mexico, Albuquerque, NM 87103 this is a nominal value. The actual spacing is $4.969 \mathrm{~ns}$.

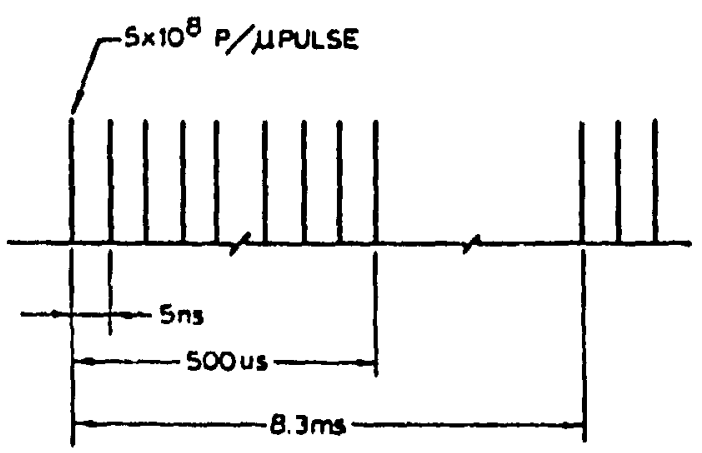

1. LAMPP Beam Pulsu Structure: Micropulses of $10^{8}$ particles at 5 nsec interval make up a 500 microsecond macropulse. Avarage current during the macropulse is $7 \mathrm{mh}$. Maciopulses occur at a 120 per second rate. epread) for given rini, circumference. If these conditions are not met, the protons will be lost from the 500 Miz rf buckets, which are phase-locked to the LAMPF micropulse repetition frequency. An overall energy acceptance of $\Delta \mathrm{B} / \mathrm{Em} 10^{-3}$ is calculated for the PSR, which includes the stability of the nominal (central) energy. This is in contrast to the operation of a synchrotron, whose tf system typically operates at a low harmonic of the circulation frequency; in this situation the monentur acceptance is usually limited by the growth of the transverse dimensions of the beam.

The beam pulse to be delivered by the linac to the PSR/WNR is prepared by means of a chopper in the $750 \mathrm{keV}$ linac injection line.

This device opens a several us long :ante in the mecropulse which isolates the PSR/WNR fulse from rino main portion. This interval parmits tice eichyard kicker magnet to turn on without spilling $800 \mathrm{MeV}$ beaw on the first magnet in the WNR transport channel. We are concerned about the transient response of the linac rt syatems to this sudden removal and replacement of the beam load, and about any consequent slewing of the output beam energy during the PSR/WNR pulse.

Such an effect might put some of the micropulses outside the energy acceptance of the PSR, but not be detectable in time-integraced measureants of the LANPF beam energy. To assess the importance of this efrect it is necessary to measure bead energy variation from micropulse to micropulse within high current macropulses which have been appropriately chopped. This could be done by direct time-resolved meanurements of the magnetic rigidity of the beam using electrostatic position sensors to detect small changes in the bending angle or by time of flight measuredents using micropulse detection in the 200 meter-long WNR heam line. These techniques would have marginal energy" resolution.

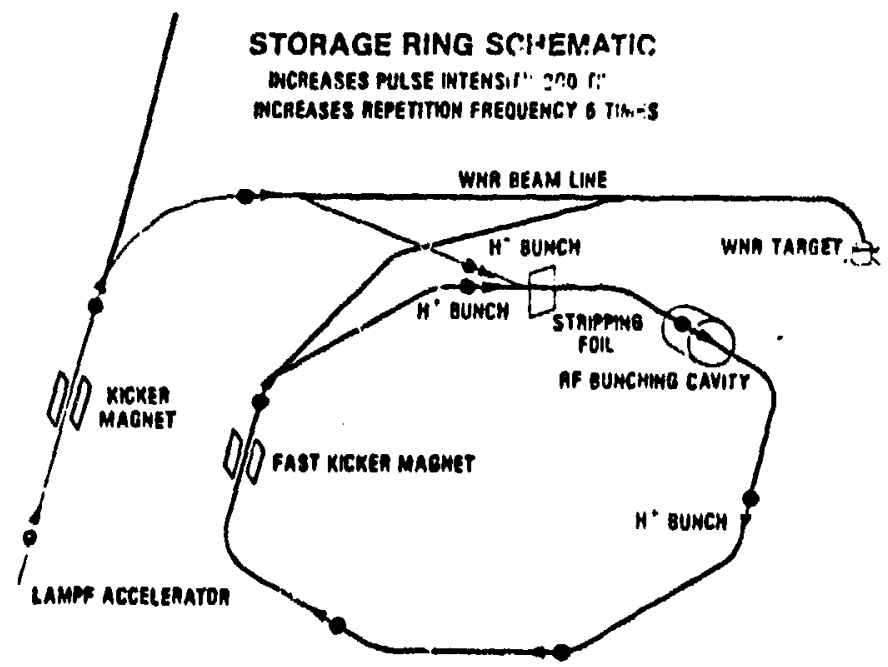

2. Short Pulse Moda of PSR Operation: LAMPF $\mathrm{H}^{-}$ion micropulses at 60 nsec intervals are accimulated in $500 \mathrm{MHz} r f$ buckets to make ix rirculating bunches of $1011800 \mathrm{MeV}$ protons. These are ejected at 1.4 maec intervals. 


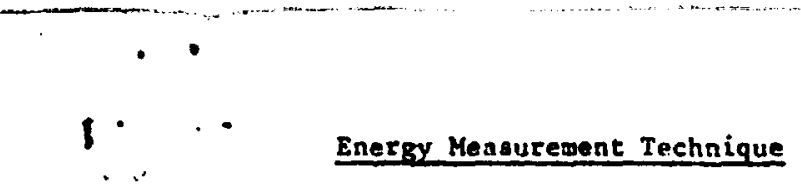

A very precise energy meastring technique is now available at LAMP. It utilizes an atomic resonance line in a manner analogous to the techniques which are conventionally employed in calibrating electrostatic accelerators. The method takes advantage of a very narrow resonance in the photodetachment spectrum of the $\mathrm{H}^{-}$ion. The low intensity $\mathrm{H}^{-}$bean which shares the accelerator with the proton beam can thus be used as a probe of the rf system reponse to chopping of the high curcent proton beam. Any transient droop in accelerating cavity voltage will be reflected by energy variation in the $\mathrm{H}^{-}$beam, which can be measured with the desired precision.

The $\mathrm{H}^{-}$br am energy is measured in the following way, using apparacus mounted in the external proton (EP) beam line in LAMPF experimental arpa $B$. The beam is directed through a pulsed photon beam from a Nd:YAC laser. By varying the laboracory angle between the ion and photon beams, the photon energy in the ion center-of-mass frame can be continuously adjusted. This method has been used for a number of sensicive atomic physics experiments at LAMPF. 3,4 The $\mathrm{H}^{-}$ ion resonance observed for bean energy measurements is the $n=2$ Feshbach photodetachment resonance. An attractive feature of the method is that this resononce, which is at $10.930 \mathrm{el}$ in the center-of-mass frame, and which is too narrow to measure, can be tuned through with both third $(3.494463 \mathrm{eV}, 0.7 \mathrm{meV}$ FWHM) and fourth (4.659284 eV, $0.9 \mathrm{meV}$ FWFM) multiples of the laser fundamental frequency. This makes possible an absolute measurement of the $\mathrm{H}^{-}$beam velocity in terws of the two different lal oratory angles (between the ion and photon beawe) for resonant photocotachment produced by the different laser haiviunics.

Figure 3 shows the essential features of the experinertal apparatus. A Q-switched Nd:YAG laser is fremien:; tripled or quadrupled to generate a 5 nsec Irig photon pilse which is directed by a series of irisms at a fixed intersection region in the ion $b \in a m$. The laboratory intersection angle $\alpha$ is set by a stepping motor and reasured by a shaft encoder to 30 microradian accuracy.

The $\mathrm{H}^{-}$ion beam passes through an upstream weak transverse magnetic field which sweeps out any fast elecirons liberated by collision with residual gas

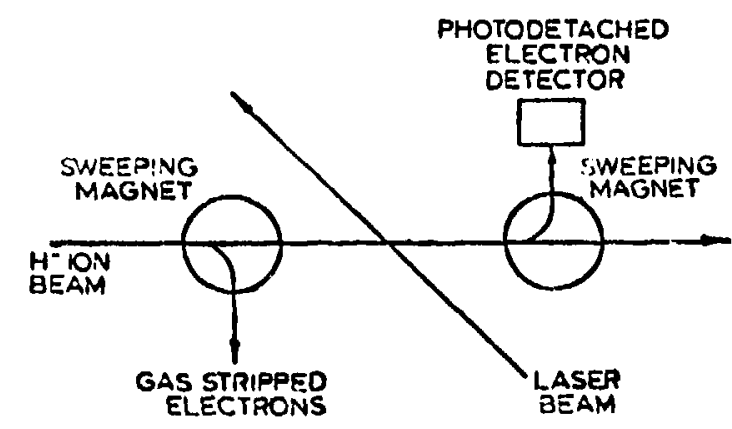

3. Experimental Schemacic Diagram: Laser beam intarects ion beam at laboratory angle $\alpha$. Ion beam is cleared of gas stripped $463 \mathrm{keV}$ electrons by upstrean sweeping magners. Downscream oweeping magnat sends phototatached $463 \mathrm{keV}$ electrons onto the detector. atoms. Photodetached electrons ( $463 \mathrm{keV}$ ) from the photon- $\mathrm{H}^{-}$ion interactions are directed onto a silicun detector by a downstream sweeping field having polarity opposite to the initial one. Pulse height analysis decerwines the number of photodetached electrons and discriminates against low energy electrons. Coincidence gating with the laser pulse gives a good signal to noise ratio. Although the laser firing jicter is $10 \mathrm{~ns}$ and the laser pulse width is $5 \mathrm{~ns}$ FWH, single micropulse timing resolution is obtained by measuring the timing of the laser pulse relative to the ion micropulse.

The following relations are basic to the experiments:

$$
E^{\prime}=\gamma E_{l a b}(I+\beta \cos \alpha)
$$

where $E^{\prime}$ is the photon energy in the center-of-mass frame, Elab is the photon energy in the laboratory frame, and $\alpha$ is the is the laboracory angle betwen the ion and photon beams, defined so that $\alpha=0$ for headon collisions. ions,

For the third harmonic photon beam and $800 \mathrm{MeV}$

$$
\begin{aligned}
& \mathrm{dE} / / \mathrm{d} \alpha=E_{1 \mathrm{ab}} Y \beta \sin \alpha-3.09 \mathrm{meV} / \mathrm{mrad} \\
& \mathrm{dE} / / \mathrm{dT}=\left(E_{1 \mathrm{ab}} / \mathrm{mc}^{2}\right)\left(1+B^{-1} \cos \alpha\right) \\
& =7.36 \mathrm{mev} / \mathrm{Mev}
\end{aligned}
$$

where $T$ is the ior kinetic energy, and

$d E^{\prime} / d E_{1 a b}=\gamma(1+B \cos \alpha)=3.13 \mathrm{meV} / \mathrm{meV}$

The angular divergence of the photon beam is $0.5 \mathrm{mrad}$, and the angular divergence of the $\mathrm{H}^{-}$ion beam is 0.2 mrad. The energy spread $\delta E_{1 a b}$ of the laser beam is $0.75 \mathrm{meV}$ and the intrinsic width of the photodetachment resonance $(n-2$ Eeshbach) is unknown, but negligible. The beam intrinsic energy spread $\delta \mathrm{T}$ can be determined from the measured resonance line width by the relation

$\left(\delta T d E^{\prime} / d T\right)^{2}=\left(\delta E_{x m s}\right)^{2}-\left(\delta E_{1 a b} d E^{\prime} / d E_{1 a b}\right)^{2}$ $-\left(\delta a d E^{\prime} / d \alpha\right)^{2}$

The last two terms on the right hand side contribute fractional linewidth equivalent to that of an $800 \mathrm{MeV}$ ion beam with a $195 \mathrm{keV}$ energy apread. By careful measurement and control of $\delta \alpha$, determination of $\delta ' \mathrm{~T}$ to $\pm 50 \mathrm{keV}$ is possible. Time-of-flight measurements made at WNR indicate a characteristic intrinaic energy spread within a micropulse of $\Delta E / E= \pm 10^{-3}$ FWhM, which is $\pm 800 \mathrm{KeV}$ for an $800 \mathrm{MeV}$ ion beam. Therefore, we can see that the photodetachment energy measurement technique delivers the desired resolution.

The centroid of the resonance lineshape can be determined to \pm 60 microradians, permitting an absolute measurement of the $\mathrm{H}^{-}$beam energy centroid to eight parts in $10^{4}( \pm 480 \mathrm{keV})$. This is marginal

(but sufficient) accuracy if it is desired to use this procedure to tune the linac for a specific energy with an accuracy of a part in $10^{3}$. 


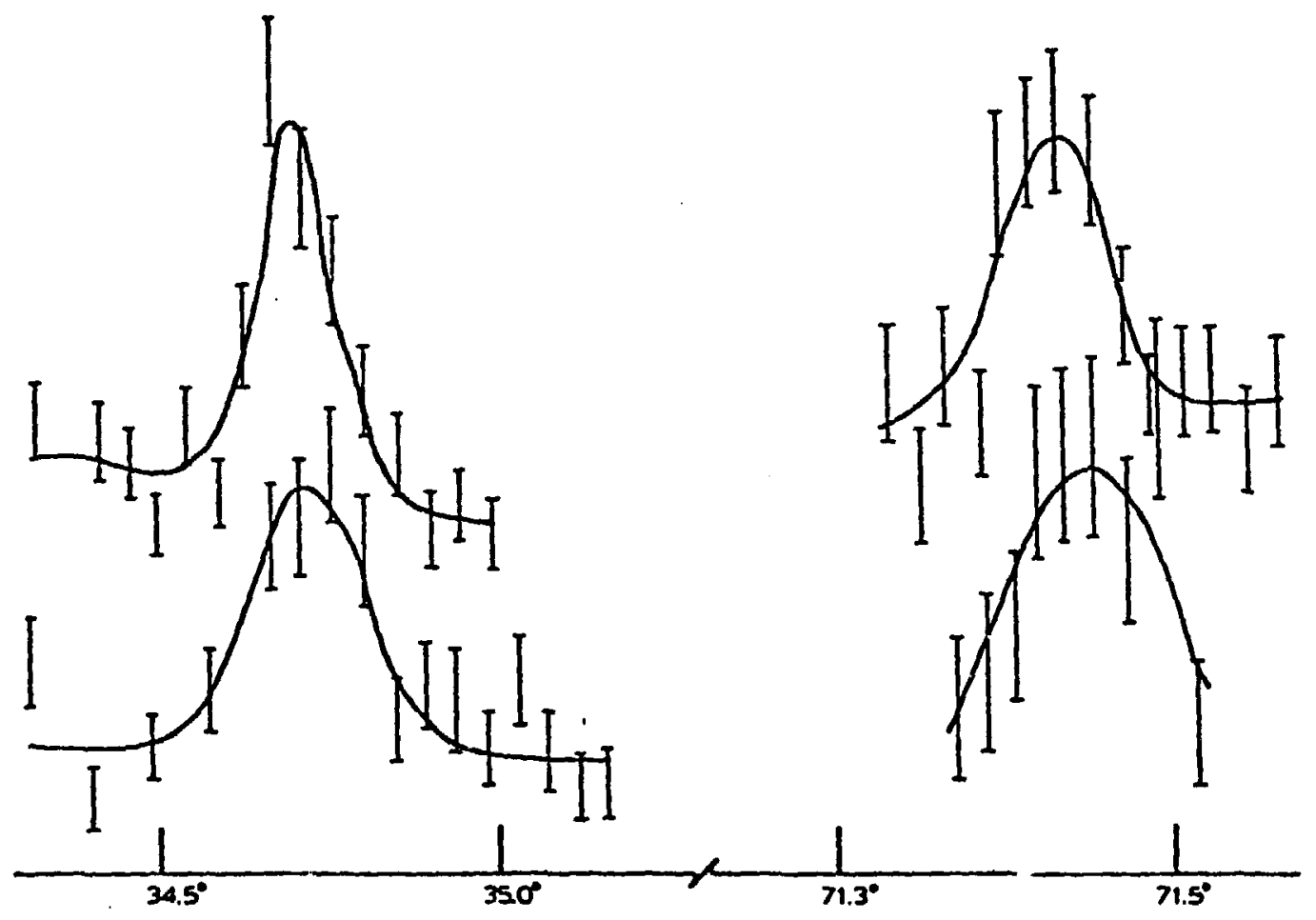

4. Preliminary Results. Third multiple regonance at 34.70 and fourth multiple resonance at 710 without time resolution or proton besm chopping.

\section{Results}

Figure 4 shows initial results obtained using the third and fourth multiples of the laser fundamental frequency marte on an unchopped beaw at one point in the macropulse. The photodetactment cross section peaks correspond to ion beam energies of $797.63 \mathrm{MeV}$ $\pm 0.36 \mathrm{MeV}$ and $798.56 \mathrm{MeV} \pm 0.35 \mathrm{MeV}$ respectively. which is consistent with the nowinal LAMPE beam energy of $795 \mathrm{MeV}$ to $800 \mathrm{MeV}$. The linewidchs correspond to FWAM energy spreads of $1.4 \mathrm{MeV}$ and $1.8 \mathrm{MeV}$, in agreement with estimates of the energy spread in the LAMPE beali made from transit time broadening of micropul ses in tite WNR beamline.
The data for the third and fourth laser frequency multiples were taken a week apart and thus cannot be used to make an absolute measurement. More extensive and detailed measurements, including time-resolution, are being made

\section{References}

1. R. K. Cooper and G. P. Lawrence, "Design of the WNR Proton Storage Ring Lattice", Proc. 1977 Particle Accelerator Conference, IEEE Trans. NS -24, 1037 (1977).

2. D. W. Hudgings, "Neutral Beam Injection for a Proton Storage Ring", Proc. 1979 Particle Accelerator Conf.

3. P.A.M. Gram, et al., "Effect of an Electric Field upon Regonances in the $\mathrm{H}^{-}$Ion", Phys. Rev. Lett. 40,10 (1978).

4. H. C. Bryant, et al., "Observations of Resonancss Near $1 \mathrm{LeV}$ in the Photodetachent Crosg-Section, $\dot{b}$ the $H^{-}$Ion", Phys. Rev. Lett. 38, 228 (1977). 